BULLETIN (New Series) OF THE

AMERICAN MATHEMATICAL SOCIETY

Volume 50, Number 4, October 2013, Pages 675-680

S 0273-0979(2013)01397-1

Article electronically published on January 17, 2013

Real solutions to equations from geometry, by Frank Sottile, University Lecture Series, Vol. 57, American Mathematical Society, Providence, Rhode Island, 2011, x+200 pp., ISBN 978-0-8218-5331-3, List Price: US $\$ 47$, Member Price US $\$ 37.60$

Real algebraic geometry studies real algebraic sets, i.e., real-number solutions to algebraic equations with real-number coefficients, and mappings between them (in particular, real polynomial mappings).

The history of real algebraic geometry goes back to ancient Greece. In the third century BCE, Archimedes and Apollonius systematically studied problems on conic sections [17, and also introduced the use of coordinates [8].

During the last two hundred years the real algebraic geometry developed into a rich discipline with deep and sometimes surprising connections to semialgebraic geometry, real algebra, logics (especially, o-minimality theory and model theory), real analytic geometry, the theory of moment problems, convex optimization, the theory of quadratic forms, valuation theory, and other subjects.

One of the first results in real algebra is the famous Hilbert's theorem [13] on ternary quartics that claims that any real positive degree four polynomial in three variables is a sum of three squares. The 17th Hilbert problem asks if any positive rational function is represented by a sum of squares. The problem was solved by Artin [6] in 1927. The further development of real algebra and real algebraic geometry is associated with names of Tarski (whose famous quantifier elimination result [29] connects real algebraic geometry and formal logic), Pólya (his theorem on positive polynomials on a simplex [26]), Motzkin (his example of positive polynomial that is not a sum of squares of polynomials 22 ), and others.

One of the central problems of the real algebraic geometry is the topology of the set of real solutions.

In 1876 Harnack 12 proved that a genus $g$ real planar algebraic curve has at most $g+1$ ovals. (This bound on the number of components was later extended to all Betti numbers of all real algebraic sets [21,25, 30, and all semialgebraic sets [7.) A curve with the maximal possible number of ovals is called an $M$-curve. The first part of the 16th Hilbert problem asks about the mutual positions of ovals of $M$-curves. Several years later V. Ragsdale formulated a conjecture on the oval arrangement for an $M$-curve [27]. Efforts to prove the Ragsdale conjecture stimulated works by V. Arnold [5], D. Gudkov and G. Utkin [11, O. Viro [32 35], V. Kharlamov [16, and others until it was disproved in 1970s by I. Itenberg [15].

Real algebraic varieties with given topological properties were studied in papers by Nash, Tognolli, Akbulut, King, Finashin, Mikhalkin, Kollar, Lojasiewicz, Hironaka (see, e.g., [1,4, 14, 18, 20, 24, 29, 31]), and others.

In particular, when the number of equations coincides with the number of variables, the solution is a collection of points and the only nontrivial topological characteristics is the number of real points.

2010 Mathematics Subject Classification. Primary 14P05.

(C) 2013 American Mathematical Society Reverts to public domain 28 years from publication 
The estimates of the number of real solutions (the upper and lower bounds), enumerative real algebraic geometry, and examples of systems of polynomial equations (having the origin in enumerative algebraic geometry) with only real solutions are the subjects of the book by F. Sottile.

Let a system of $n$ polynomial equations in $n$ variables with real coefficients be given:

$$
F_{i}\left(x_{1}, \ldots, x_{n}\right)=0, i=1, \ldots, n .
$$

Here, $F_{i}\left(x_{1}, \ldots, x_{n}\right)=\sum_{I} a_{i}^{I} x^{I}$ is a real polynomial in $n$ variables $x_{1}, \ldots, x_{n}$, $a^{I} \in \mathbb{R}$, where $I \in \mathbb{Z}_{\geq 0}^{n}$ is a multiindex.

Even the existence of a real solution is often hard to abjudicate. The situation is much simpler if we consider complex solutions in $\mathbb{C}^{n}$. Then, at least we can guarantee the existence of a solution for a generic system. Sometimes even the number of complex solutions can be estimated.

As a trivial example we can recall that the fundamental theorem of algebra claims that a polynomial in one variable of degree $d$ has exactly $d$ complex solutions counting multiplicities. Moreover, the famous Bezout theorem states that two algebraically independent polynomials of two variables $x$ and $y$ of degrees $d_{1}$ and $d_{2}$ have $d_{1} d_{2}$ roots counting multiplicities and roots at infinity. To the contrary, the real quadratic polynomial $x^{2}-1=0$ has two real roots while $x^{2}+1=0$ has none.

In middle school we learned the method of finding the number of real roots of a real quadratic polynomial $R(x)=x^{2}+p x+q$. The recipe is known from the time of classical Greece, ancient India, and Babylon. Equation $R(x)=0$ has real roots if and only if the discriminant $D=p^{2}-4 q \geq 0$. If the discriminant is zero, the solution is real and unique; otherwise, there are no real solutions (two roots form a complex conjugated pair).

This provides a complete characterization of the number of solutions in terms of the coefficients of polynomial. This is a dream of mathematicians studying bounds of the number of real solutions. For one univariate polynomial of higher degree, the upper bound for the number of positive roots can be obtained by Descartes' rule of signs and a more precise answer by Sturm theorems [28].

The case of systems of $n$ polynomial equations in $n$ variables does not allow, in general, such a nice answer. The number of complex solutions is described by Kushnirenko's theorem $(n ! \cdot \operatorname{Volume}(\Delta)$, where $\Delta$ is the Newton polytope of the system). Note that Kushnirenko's theorem gives an exact number of roots for generic polynomial systems and an upper bound for other cases.

The number of real solutions is estimated roughly not by the degrees of polynomials in a system but rather by the number of nontrivial monomials in their expressions. The most general result is the famous Khovanskii's fewnomial bound:

Theorem 1. A system of $n$ real polynomials in $n$ variables involving $1+\ell+$ $n$ distinct monomials has fewer than $2^{\left(\begin{array}{c}\ell+n \\ 2\end{array}\right)} \cdot(n+1)^{\ell+n}$ nondegenerate positive solutions.

This bound is far from being sharp. Despite the amazing results obtained by the fewnomial method, its limitations are characterized by the classical Maxwell conjecture: the electrostatic field in $\mathbb{R}^{3}$ generated by three fixed unit charges has only four or two equilibria. It is easy to check that the system of polynomial equations with roots at equillibria has much larger number of complex solutions. 
However, no more than four solutions are real. This innocent looking conjecture is still out of reach by modern methods. The best upper bound 12 is obtained in [10.

The lower bounds are interesting because they demonstrate the existence of solutions. The existence is especially interesting for problems of enumerative real algebraic geometry, such as, for instance, computing the number of real planar algebraic curves of degree $d$ passing through $3 d-1$ generic real points. It is clear that the answer depends on the configuration of points. Therefore it is interesting to find out if such curve exists for any configuration. The Welschinger invariant 36 gives a nontrivial lower bound for the minimal number of such curves. In particular, the nonzero Welschinger invariant demonstrates that such a real curve exists.

Recalling the notion of $M$-curves, we ask the following question: "What can we say about the configuration of points such that all corresponding curves are real?" A similar question was asked in the 1980s by W. Fulton about Schubert calculus.

Grassmannian (or Grassmann space) $G_{k}(n)$ is the set of all $k$-dimensional vector subspaces of $n$-dimensional vector space. A $k$-subspace (or Grassmann element) is called real if it is invariant with respect to complex conjugation.

This set has a structure of projective algebraic manifold. The standard embedding into a projective space is given by Plücker coordinates. Namely, choose a standard basis in $\mathbb{C}^{n}$. Choose $k$ independent vectors and write them as rows of a $k \times n$ matrix. The determinant of the minor of maximal size $k$ formed by columns $i_{1}<i_{2}<\cdots<i_{k}$ of the matrix is called the Plücker coordinate $x_{i_{1}, \ldots, i_{k}}$. Note that other choices of basis or collection of vectors lead to the simultaneous multiplication of all Plücker coordinates by the same constant. Therefore, all Plücker coordnates define the embedding of $G_{k}(n)$ in projective space. There is a standard cell decomposition of $G_{k}(n)$ into Schubert cells defined as follows. Its closure, called Schubert variety, is a certain subvariety of Grassmannian, usually with singular points, which consists of the $k$-dimensional subspaces $V$ such that $\operatorname{dim}\left(V \cap F^{a_{j}}\right) \geq j$ for $j=1,2, \ldots, k$, where $F^{\bullet}=\left\{F^{1} \subset F^{2} \subset \cdots \subset F^{n}=\mathbb{C}^{n}\right\}$ is a certain flag of subspaces in $\mathbf{C}^{n}, \operatorname{dim} F^{j}=j$, and $0<a_{1} \leq a_{2} \leq \cdots \leq a_{k} \leq n$. Schubert varieties form an integer basis in homologies of the Grassmannian. Schubert calculus expresses homological intersection of Schubert cycles as a linear combination of other Schubert cycles.

Intersection of Schubert cycles of codimensions $c_{1}, \ldots, c_{m}$ such that $c_{1}+\cdots+$ $c_{n}=\operatorname{dim} G_{k}(n)=k \times(n-k)$ is a zero-dimensional cycle, i.e., is a collection of points. Each Schubert cycle can be realized as a concrete algebraic subvariety after a choice of a complete reference flag $F^{\bullet}$. Then the homology class of the intersection of corresponding Schubert cycles is represented by a concrete set of points. The following question was originally asked by W. Fulton: Is it possible to find a collection of real Schubert varieties (i.e., the corresponding reference flags are real) such that all intersection points are real too?

The conjecture formulated about 1985 by B. Shapiro and the author of this review claims that if one takes the real rational normal curve and chooses $m$ oscullating flags as reference flags, then all intersection points of the corresponding Schubert cycles are real.

This conjecture was recently proved by E. Mukhin, V. Tarasov, and A. Varchenko [23. The proof uses the theory of polynomial $s l_{2}$-representation theory and Bethe ansatz. 


\section{CONTENT OF THE BOOK}

Frank Sottile is one of the leading experts in the field of real algebraic geometry, intersection theory, and Schubert calculus. He created a school of experimental mathematics checking numerically many conjectures in real enumerative geometry. This requires not only a deep knowledge of algebraic geometry but also the skills and ingenuity to develop sophisticated numerical computational algorithms. The observations obtained by the experimental data were generalized to many more conjectures (in particular, the secant conjecture and the monotone conjecture).

His book brings us to the frontier of the research in this area. It discusses in depth the estimates for the number of real solutions of a system of polynomial equations and a totally real intersection of Schubert cycles in a Grassmannian.

In the introductory part of the book, Sottile formulates classical rules for counting real zeroes of univariate polynomials, such as Descartes's rule of signs and the Sturm theorem. Then he gives accounts of more modern theories, such as Kushnirenko's theorem computing the number of complex zeros of the system in terms of the volume of the Newton polytopes. An upper bound is obtained in the theory of fewnomials in a series of works by Khovansky and coauthors. The rest of the introduction is devoted to the Shapiros' conjecture formulated in terms of the number of zeros of Wronsky map. The Wronskian of univariate polynomials $f_{1}(t), \ldots, f_{m}(t)$ is the determinant

$$
\operatorname{Wr}\left(f_{1}, \ldots, f_{m}\right):=\operatorname{det}\left(\left(\frac{d}{d t}\right)^{i-1} f_{j}(t)\right)_{i, j=1, \ldots, m} .
$$

The Wronski map,

$$
\text { Wr }: \operatorname{Gr}\left(m, \mathbb{C}_{m+p-1}[t]\right) \rightarrow \mathbb{P}\left(\mathbb{C}_{m p}[t]\right) \simeq \mathbb{P}^{m p},
$$

where $\operatorname{Gr}\left(m, \mathbb{C}_{m+p-1}[t]\right)$ is the Grasmannian of $m$-dimensional subspaces of the linear space $\mathbb{C}_{m+p-1}[t]$ of complex polynomials of degree $m+p-1$ in the variable $t$, and $\mathbb{P}\left(\mathbb{C}_{m p}[t]\right)$ is the projective space of complex polynomials of degree at most $m p$, which has dimension $m p$, equal to the dimension of the Grassmannian. The Wronski map is surjective, and the general polynomial $\Phi \in \mathbb{P}^{m p}$ has

$$
\sharp_{m p}:=\frac{1 ! 2 ! \cdots(m-1) !(m p) !}{m !(m+1) ! \cdots(m+p-1) !}
$$

preimages under the Wronski map.

The Shapiros' conjecture can be reformulated as follows: If the polynomial $\Phi \in$ $\mathbb{P}^{m p}$ has only real zeros, then every preimage in $\mathrm{Wr}^{-1}(\Phi)$ is real. Moreover, if $\Phi$ has $m p$ simple real zeros, then there are $\sharp_{m p}$ real points in $\mathrm{Wr}^{-1}(\Phi)$.

In the first part, the book gives a detailed account on the upper and lower bounds.

The second part of the book describes the proof of the Shapiros' conjecture.

Despite its simple formulation, the proof of this conjecture took more than twenty years. The particular case of $\operatorname{Gr}(2, n)$ ( or $\operatorname{Gr}(n-2, n)$ ) was proved by A. Gabrielov and A. Eremenko 9] in 2002 in the following form: any degree $n$ rational function $P(x) / Q(x)$ with only real critical points can be made real by the corresponding Möbius transform. The proof of this statement is based on the beautiful theory of nets in the disk.

The complete proof was published by E. Mukhin, V. Tarasov, and A. Varchenko [23] in 2009. 
The last section of the book is devoted to generalizations of the Shapiros' conjecture and open problems, such as the monotone conjecture, the secant conjecture, and analogues of the Shapiros' conjecture for orthogonal Grassmannians and Lagrangian Grassmannians.

\section{REFERENCES}

[1] Selman Akbulut, Real algebraic structures, Proceedings of Gökova Geometry-Topology Conference 2005, Gökova Geometry/Topology Conference (GGT), Gökova, 2006, pp. 49-58. MR2282009 (2007j:14087)

[2] S. Akbulut and H. King, Submanifolds and homology of nonsingular real algebraic varieties, Amer. J. Math. 107 (1985), no. 1, 45-83, DOI 10.2307/2374457. MR778089 (86m:14017)

[3] Selman Akbulut and Henry C. King, The topology of real algebraic sets with isolated singularities, Ann. of Math. (2) 113 (1981), no. 3, 425-446, DOI 10.2307/2006992. MR621011 (83b:58003)

[4] S. Akbulut and H. King, All knots are algebraic, Comment. Math. Helv. 56 (1981), no. 3, 339-351, DOI 10.1007/BF02566217. MR639356 (83m:57005)

[5] V. I. Arnol'd, The situation of ovals of real plane algebraic curves, the involutions of fourdimensional smooth manifolds, and the arithmetic of integral quadratic forms, Funkcional. Anal. i Priložen. 5 (1971), no. 3, 1-9 (Russian). MR0286790 (44 \#3999)

[6] E. Artin, Uber die Zerlegung definiter Funktionen in Quadrate, Abh. Math. Sem. Univ. Hamburg, 5 (1927), 85-99.

[7] S. Basu, On bounding the Betti numbers and computing the Euler characteristic of semialgebraic sets, Discrete Comput. Geom. 22 (1999), no. 1, 1-18, DOI 10.1007/PL00009443. MR.1692627 (2000d:14061)

[8] J. Dieudonné, The historical development of algebraic geometry, Amer. Math. Monthly 79 (1972), 827-866. MR0308117 (46 \#7232)

[9] A. Eremenko and A. Gabrielov, Rational functions with real critical points and the B. and M. Shapiro conjecture in real enumerative geometry, Ann. of Math. (2) 155 (2002), no. 1, 105-129, DOI 10.2307/3062151. MR1888795 (2003c:58028)

[10] A. Gabrielov, D. Novikov, and B. Shapiro, Mystery of point charges, Proc. Lond. Math. Soc. (3) 95 (2007), no. 2, 443-472, DOI 10.1112/plms/pdm012. MR2352567 (2008g:31008)

[11] D. A. Gudkov and G. A. Utkin, The topology of curves of degree and surfaces of degree, Uchen. Zap. Gorkov. Univ., vol. 87, 1969 (Russian), English transl., Transl. AMS 112.

[12] Axel Harnack, Ueber die Vieltheiligkeit der ebenen algebraischen Curven, Math. Ann. 10 (1876), no. 2, 189-198, DOI 10.1007/BF01442458 (German). MR 1509883

[13] David Hilbert, Ueber die Darstellung definiter Formen als Summe von Formenquadraten, Math. Ann. 32 (1888), no. 3, 342-350, DOI 10.1007/BF01443605 (German). MR.1510517

[14] Heisuke Hironaka, Resolution of singularities of an algebraic variety over a field of characteristic zero. I, II, Ann. of Math. (2) 79 (1964), 109-203; ibid. (2) 79 (1964), 205-326. MR0199184(33 \#7333)

[15] Ilia Itenberg, Contre-examples à la conjecture de Ragsdale, C. R. Acad. Sci. Paris Sér. I Math. 317 (1993), no. 3, 277-282 (French, with English and French summaries). MR.1233426 (94f:14050)

[16] V. M. Kharlamov, New congruences for the Euler characteristic of real algebraic manifolds, Funkcional. Anal. i Priložen. 7 (1973), no. 2, 74-78 (Russian). MR0331403 (48 \#9736)

[17] Morris Kline, Mathematical thought from ancient to modern times, Oxford University Press, New York, 1972. MR0472307 (57 \#12010)

[18] János Kollár, The Nash conjecture for threefolds, Electron. Res. Announc. Amer. Math. Soc. 4 (1998), 63-73 (electronic), DOI 10.1090/S1079-6762-98-00049-3. MR1641168 (99k:57038)

[19] S. Lojasiewicz, Triangulation of semi-analytic sets, Ann. Scuola Norm. Sup. Pisa (3) 18 (1964), 449-474. MR0173265 (30 \#3478)

[20] G. Mikhalkin, Blowup equivalence of smooth closed manifolds, Topology 36 (1997), no. 1, 287-299, DOI 10.1016/0040-9383(95)00062-3. MR.1410476 (98f:57046)

[21] J. Milnor, On the Betti numbers of real varieties, Proc. Amer. Math. Soc. 15 (1964), 275-280. MR0161339(28 \#4547) 
[22] T. S. Motzkin, The arithmetic-geometric inequality, Inequalities (Proc. Sympos. WrightPatterson Air Force Base, Ohio, 1965), Academic Press, New York, 1967, pp. 205-224. MR.0223521 (36 \#6569)

[23] Evgeny Mukhin, Vitaly Tarasov, and Alexander Varchenko, The B. and M. Shapiro conjecture in real algebraic geometry and the Bethe ansatz, Ann. of Math. (2) 170 (2009), no. 2, 863-881, DOI 10.4007/annals.2009.170.863. MR2552110(2011b:17065)

[24] John Nash, Real algebraic manifolds, Ann. of Math. (2) 56 (1952), 405-421. MR0050928 $(14,403 \mathrm{~b})$

[25] I. G. Petrovskiĭ and O. A. Oleŭnik, On the topology of real algebraic surfaces, Izvestiya Akad. Nauk SSSR. Ser. Mat. 13 (1949), 389-402 (Russian). MR0034600 (11,613h)

[26] G. Pólya, Über positive Darstellung von Polynomen Vierteljschr, Naturforsch. Ges. Zürich 73 (1928) 141-145, in: R. P. Boas (Ed.), Collected Papers Vol. 2, MIT Press, Cambridge, MA, 1974, pp. 309-313.

[27] V. Ragsdale, On the arrangement of the real branches of plane algebraic curves, Amer. J. Math. 28 (1906), no. 4, 377-404, DOI 10.2307/2370070. MR1505999

[28] J. C. F. Sturm, Mémoire sur la résolution des équations numériques, Mémoires divers présenteés par des savant étrangers à l'Académie Royale des Sciences de l'Institut de France, sciences mathématiques et physiques, 6 (1835) 273-318.

[29] A. Tarski, A decision method for elementary algebra and geometry, Rand. Corp.. 1948; UC Press, Berkeley, 1951, Announced in Ann. Soc. Pol. Math. 9 (1930, published 1931) 206-207; and in Fund. Math. 17 (1931) 210-239.

[30] René Thom, Sur l'homologie des variétés algébriques réelles, Differential and Combinatorial Topology (A Symposium in Honor of Marston Morse), Princeton Univ. Press, Princeton, N.J., 1965, pp. 255-265 (French). MR0200942 (34 \#828)

[31] A. Tognoli, Su una congettura di Nash, Ann. Scuola Norm. Sup. Pisa (3) 27 (1973), 167-185. MR.0396571 (53 \#434)

[32] O. Ya. Viro, Gluing of plane real algebraic curves and constructions of curves of degrees 6 and 7, Topology (Leningrad, 1982), Lecture Notes in Math., vol. 1060, Springer, Berlin, 1984, pp. 187-200, DOI 10.1007/BFb0099934. MR770238(87i:14029)

[33] O. Ja. Viro, Curves of degree 7, curves of degree 8 and the Ragsdale conjecture, Dokl. Akad. Nauk SSSR 254 (1980), no. 6, 1306-1310 (Russian). MR592496 (82e:14067)

[34] O. Ya. Viro, Gluing of plane real algebraic curves and constructions of curves of degrees 6 and 7, Topology (Leningrad, 1982), Lecture Notes in Math., vol. 1060, Springer, Berlin, 1984, pp. 187-200, DOI 10.1007/BFb0099934. MR770238 (87i:14029)

[35] O. Ya. Viro, Real plane algebraic curves: constructions with controlled topology, Algebra i Analiz 1 (1989), no. 5, 1-73 (Russian); English transl., Leningrad Math. J. 1 (1990), no. 5, 1059-1134. MR 1036837 (91b:14078)

[36] Jean-Yves Welschinger, Invariants of real symplectic 4-manifolds and lower bounds in real enumerative geometry, Invent. Math. 162 (2005), no. 1, 195-234, DOI 10.1007/s00222-0050445-0. MR2198329(2006i:53123)

Michael Shapiro

Michigan State University 